\title{
Analysis and Implementation of an Artificial Homeostatic Hormone System: A First Case Study in Robotic Hardware
}

\author{
Jürgen Stradner*, Heiko Hamann*, Thomas Schmickl*, Karl Crailsheim*
}

\begin{abstract}
One of the prominent challenges in mobile robotics is to develop control methodologies that allow the adaptation to dynamic and unforeseen environments. The classic approach of hand-coded controllers is very efficient for well-defined tasks and specific environments but poor in adapting to changing environmental conditions. One alternative approach is the application of evolutionary algorithms which need, in turn, easily evolvable representations of controllers. In this paper, we investigate one promising approach of an artificial hormone system as a control paradigm which is believed to be easily optimized by evolutionary processes. In a first step of this research, we focus on the simple task of collision avoidance. We present a brief mathematical analysis of this controller approach and an implementation of the controller on a mobile robot to check the feasibility in principle of our approach. The task is successfully accomplished and we conclude with a discussion of the hormone dynamics in the robot.
\end{abstract}

\section{INTRODUCTION}

A main challenge in mobile robotics is the strict linkage of the robot's performance to the environment it is designed for. Changes in the environment may affect the effectiveness of the robot controller essentially. All kinds of variations in the sensory input as well as changed effects of the actuators in different environments cannot be envisaged in the control design phase. The lack of abilities of mobile robots to adapt to new environments constricts their field of applications.

One way out of this dilemma might be evolutionary robotics [1]. Simulation studies about learning in artificial neural networks showed that agents can be selected by their ability to develop a predisposition to adapt and learn [2]. The aim of this research project is to develop a representation of robot controllers, the Artificial Homeostatic Hormone Systems (AHHS), that allow the evolution of controllers with high evolving abilities. In a first step, we have developed a controller representation that is believed to lead to smooth fitness landscapes because it is defined by (in principle) continuous values [3]. Often a small change of these parameters of the AHHS causes also small changes in the behavior of the controlled agent. This property is important for the planned application of evolutionary algorithms. Mutations of the configuration of the AHHS will lead to small improvements or small declines in the fitness allowing an easy gradient ascent in the fitness landscape, see for example [4]. Furthermore, the AHHS seems to have a potential of being easily adaptable to dynamic environments.

There are other studies also suggesting hormone-inspired control for autonomous robots: [5] describes a hormone-

\footnotetext{
* Department of Zoology, Karl-Franzens University Graz, 8010 Graz,
} Universitätsplatz 2, Austria, juergen. stradner@uni-graz . at

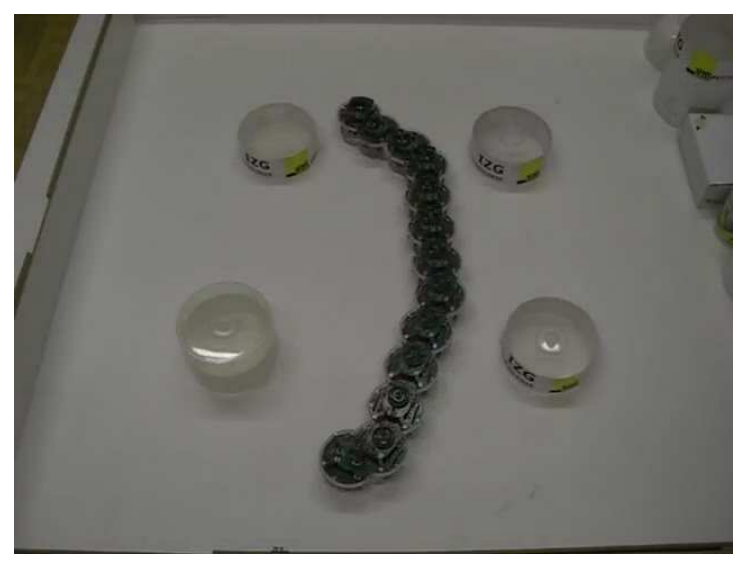

Fig. 1. Stroboscopic image of the arena with a moving e-puck robot and obstacles.

based system, in which hormones are understood as messages that are routed among the agents. In our AHHS, hormones are modeled like chemical substances, which flow through a virtual (robotic) organism. This controller is inspired by real organisms and reflects their information processing mechanisms that involve hormones and secondmessengers. Complex behaviors are performed by the controlled automata (robots) as they pursue homeostatic control of internal (virtual) hormone values, which are disturbed by external sensory stimuli.

The design and analysis of this big vision of the AHHS as described above are a long term project. In this paper, a first mathematical analysis and the first implementation of such a controller in a real robot is reported to prove the general feasibility of this approach.

\section{THE AHHS CONTROLLER}

\section{A. The controller}

This bio-inspired controller is generally described in [3]. The basic idea of an AHHS is inspired by secondmessengers, which act like inter-cellular hormones in evolutionary "simple" uni-cellular organisms like protozoa and bacteria. The basic principle of an AHHS is that robot sensors can trigger the release of virtual hormones. The inner body of the robot is spatially represented by compartments, and each sensor excretes only a specific "hormone" into its associated compartments. These hormones then decay over time, and diffuse to neighboring compartments. In this way the information of past sensor activation is spread throughout the whole "inner body" of the robot. Hormones interact with each other, that is, they multiply or decrease the level of 


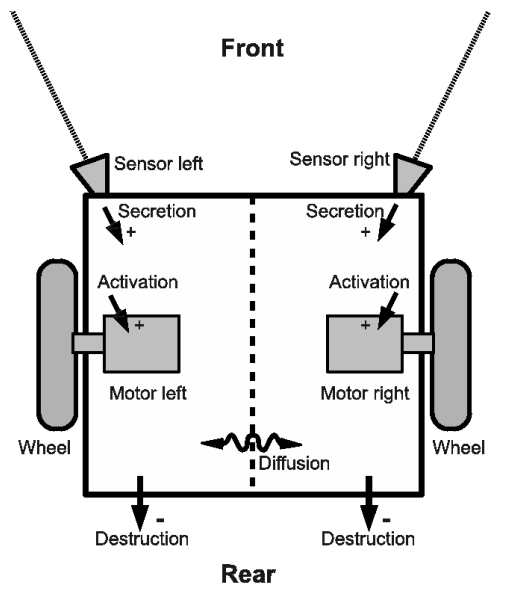

Fig. 2. Basic structure of our analyzed AHHS controller.

other hormones. And finally, they activate actuators, which manifest the robot's behavior. As this robot behavior alters the sensor stimulation, a sensor-controller-actuator feedback loop arises which is governed by our controller such that a hormone level is kept around a homeostatic set point.

A simple example of such an AHHS controller is described as follows: A robot measures the distance to nearby obstacles by infrared sensors. Excitation of these sensors leads to the excretion of hormones reflecting "collision stress". This alters the activation of motors, such that the robot performs an avoidance (turning) behavior until the obstacle is far away and the concentration of the hormone decays. Thus, the robot achieved the intrinsic homeostatic set point again and continues its normal behavior. We claim that such a system provides a smooth parameter landscape to allow artificial evolution that adapts the sensor-to-hormone rules, the rules of hormone-to-hormone interaction, and the hormone-toactuator rules.

In this paper, we chose a very simple compartment topology. In our experiments, the agent's interior is virtually separated in two compartments: a left and a right compartment. Each compartment contains one proximity sensor to the front (about $45^{\circ}$ shifted to the side) and one actuator (locomotion of a wheel). Fig. 2 shows the structure of our basic AHHS system, which is described, analyzed, and implemented in this article. Both compartments contain hormones that diffuse between the two compartments. The hormone concentrations are influenced by five parameters:

- base emission rate $\alpha$ : unconditioned emission of hormone per time step

- decay rate $\lambda$ : rate of reduction of the hormone per time step

- diffusion coefficient $D$ : the fraction of the hormone difference that is transferred from the compartment with a higher hormone concentration to the other compartment

- sensor dependent emission rate $\gamma$ : emission of hormone per time step depending on the current sensor value

- actuator scale factor $\sigma$ : the influence of the hormone to the actuator control value (linear scaling)
TABLE I

PARAMETERS OF THE CONTROLLER WITH ONE HORMONE

\begin{tabular}{|l|c|}
\hline parameter & value \\
\hline base emission rate $\alpha$ & $1001 /[$ time unit] \\
decay rate $\lambda$ & $0.91 /$ [time unit] \\
diffusion coefficient $D$ & $0.031 /[$ time unit] \\
sensor dependent emission rate $\gamma$ & $101 /[$ time unit] \\
actuator scale factor $\sigma$ & 0.5 \\
\hline
\end{tabular}

Each hormone is defined by its own set of parameters.

The general update rule for time step $t+1$ based on time step $t$ in form of a difference equation for a hormone $H(t)$ is given by

$$
\frac{\Delta H}{\Delta t}=\alpha-\lambda H(t)+\gamma S(t)+D\left(H_{\text {neighbor }}(t)-H(t)\right),
$$

for a sensor value $S(t)$ and for the hormone value in a neighboring compartment $H_{\text {neighbor }}(t)$. Information about a sensor value of the other compartment is only transmitted via diffusion. The actuator control value $a(t)$ is, in general, set by each hormone $H_{i}$ via

$$
a(t+\Delta t)=\sum_{i=1}^{N} \sigma_{i} H_{i}(t),
$$

for $N$ hormones and the actuator scale factor $\sigma_{i}$ of hormone $i$.

In the paper at hand, we analyze the behavior of two different types of hormone controllers that are presented in the following. These two controllers have been investigated by simulation and sensitivity analysis in [3], now we implement them into real robot hardware. In the first type only one hormone $H_{d}$ is used to control the behavior of the agent. The base emission rate $\alpha$ of this hormone is responsible for the default velocity of the agent when there is no obstacle detected. A detection of an obstacle by a sensor leads to an increase of hormone $H_{d}$ value due to the sensor dependent emission rate $\gamma$. An unilaterally increased concentration leads to a turning behavior as described above. The values of the parameters for the controller were calibrated in preliminary tests and they are listed in Tab I.

The second controller type uses two additional hormones to control the robot. The first hormone $H_{d}$ is identical to the one hormone described above except that the sensor dependent emission rate $\gamma$ is fixed to 0 . This makes $H_{d}$ responsible for the default velocity which is set by the base emission rate $\alpha$ of the hormone. The two variable hormones responsible for the steering of the agent are called left hormone $H_{l}$ and right hormone $H_{r}$. The left hormone is emitted in the left compartment when the left sensor detects obstacles and vice versa. An increased value of the left hormone $H_{l}$ leads to an increase in the actuator control value $a^{\text {left }}$ on the same side. The left hormone $H_{l}$ reaches the right compartment by diffusion. In this opposite compartment the left hormone $H_{l}$ decreases the actuator control value $a^{\text {right }}$. The right hormone $H_{r}$ is emitted due to activation by the 
TABLE II

PARAMETERS OF THE CONTROLLER WITH THREE HORMONES

\begin{tabular}{|l|c|}
\hline parameter & value \\
\hline base emission rate $\alpha$ & $1001 /[$ time unit] \\
decay rate $\lambda$ & $0.51 /$ [time unit] \\
diffusion constant $D$ & $0.21 /$ [time unit] \\
sensor dependent emission rate $\gamma$ & $101 /[$ time unit] \\
actuator scale factor $\sigma_{\text {own }}$ & 0.1 \\
actuator scale factor $\sigma_{\text {opposite }}$ & -0.3 \\
\hline
\end{tabular}

right sensor at the right compartment. It increases the right actuator control $a^{\text {right }}$ but decreases the left actuator $a^{\text {left }}$ by reaching the opposite compartment due to diffusion. Thus, the velocity of the robot might drop beneath the default velocity that is set by hormone $H_{d}$.

The actuator scale factor $\sigma$ is different depending on the hormone and the compartment. The left hormone $H_{l}$ has an actuator scale factor of $\sigma_{l}=\sigma_{\text {own }}=0.1$ at the left compartment and $\sigma_{r}=\sigma_{\text {opposite }}=-0.3$ at the right compartment. This is defined vice versa for the right hormone $H_{r}$. The other parameters are identical for both hormones and both compartments, see Tab. II.

\section{ANALYSIS OF THE CONTROLLER}

Before the controllers were implemented on the robot the controller using one hormones was modeled and analyzed mathematically for the robot topology as described above. Using a mathematical model we obtained a good overview of the behavior classes that are defined by the controller.

We chose a system of difference equations instead of differential equations to model the agent because it resembles the processes in the robot more closely (discrete time). The agent's position is given by $\mathbf{x}$ and updated by

$$
\frac{\Delta \mathbf{x}}{\Delta t}=\left(\begin{array}{c}
\cos \phi \\
\sin \phi
\end{array}\right) v
$$

for heading $\phi$ and a constant velocity $v>0$. The assumption of a constant velocity is a simplification because the hormone controller could, in principle, cause varying velocities. However, most of these changes take place during turning processes changing mainly the angular velocity in this work. The velocity $v$ also incorporates the base emission rate $\alpha$ of $H_{d}$. The changes of the heading are given by

$$
\frac{\Delta \phi}{\Delta t}=\left(H_{d}^{\text {right }}(t)-H_{d}^{\text {left }}(t)\right) \theta
$$

for a concentration of the hormone $H_{d}$ in the right compartment $H_{d}^{\text {right }}$, the left compartment $H_{d}^{\text {left }}$, and a parameter $\theta$ that defines the intensity of the turns related to the spatial difference of the hormone. The dynamics of the hormone is given by

$$
\begin{aligned}
& \frac{\Delta H_{d}^{\mathrm{left}}}{\Delta t}=S_{l}(t)-\lambda H_{d}^{\mathrm{left}}(t)+D\left(H_{d}^{\mathrm{right}}(t)-H_{d}^{\mathrm{left}}(t)\right), \\
& \frac{\Delta H_{d}^{\mathrm{right}}}{\Delta t}=S_{r}(t)-\lambda H_{d}^{\mathrm{right}}(t)+D\left(H_{d}^{\mathrm{left}}(t)-H_{d}^{\mathrm{right}}(t)\right),
\end{aligned}
$$

TABLE III

PARAMETERS OF THE MATHEMATICAL MODEL

\begin{tabular}{|l|c|}
\hline parameter & value \\
\hline decay $\lambda$ & $0.11 /[$ time unit] \\
diffusion $D$ & $0.11 /[$ time unit] \\
steering intensity $\theta$ & 0.01 \\
velocity $v$ & 0.05 [space unit]/[time unit] \\
sensor range $r$ & 2.0 [space unit] \\
wall base point $\mathbf{w}_{1}$ & $(0,0)$ \\
wall direction $\mathbf{w}_{2}$ & $(1,1)$ \\
initial agent position $\mathbf{x}(0)$ & $(-4,4)$ \\
initial agent direction $\phi(0)$ & $321^{\circ}$ \\
sensor angle offset $\delta$ & $45^{\circ}$ \\
\hline
\end{tabular}

for a sensor input of $S_{l}$ and $S_{r}$ from the left and the right sensor, a hormone decay rate $\lambda$, and diffusion $D$. Preparing the definition of $S$ we define two vectors $\mathbf{s}_{l}^{\text {beam }}$ and $\mathbf{s}_{r}^{\text {beam }}$ that point in the direction of the beams emitted by the sensors (e.g., imagine infrared sensors):

$$
\mathbf{s}_{l}^{\text {beam }}=\left(\begin{array}{c}
\cos (\phi(t)+\delta) \\
\sin (\phi(t)+\delta)
\end{array}\right),
$$

for the sensor offset angle $\delta$ (analog for $\mathbf{s}_{r}^{\text {beam }}$ ). Now we define the input to the controller from the left sensor $S_{l}$ which is given by the cross section of two lines:

$$
\begin{aligned}
& S_{l}(t)= \\
& \begin{cases}r-\left\|\mu(t) \mathbf{s}_{l}^{\text {beam }}\right\|, & \text { for } r<\left\|\mu(t) \mathbf{s}_{l}^{\text {beam }}\right\| \wedge \mu(t)>0 \\
0, & \text { else }\end{cases}
\end{aligned}
$$

where $\mu$ is obtained by solving (if possible)

$$
\mathbf{x}(t)+\mu(t) \mathbf{s}_{r}^{\text {beam }} \stackrel{!}{=} \mathbf{w}_{1}+\nu(t) \mathbf{w}_{2},
$$

for $\mathbf{w}_{1}$ and $\mathbf{w}_{2}$ defining the position and orientation of a wall. In Eq. 8 the distance from the agent to this cross section $\left\|\mu(t) \mathbf{s}_{l}^{\text {beam }}\right\|$ is calculated. If this distance is bigger than the sensor range the sensor cannot measure the distance and returns $S_{l}(t)=0$. If the distance is smaller than the sensor range the sensor returns a "degree of proximity" (small values for big distances, big values for small distances). $S_{r}$ is similarly defined. By Eq. 9 the cross section of the sensor beam and the wall is calculated.

This model generates four classes of agent trajectories: simple turns, overreaction turns leading to rather complex trajectories, periodical turns (circular trajectories), and quasiperiodic behavior (trajectories that are repetitive but not exactly periodic). The standard parameters that were used in the following runs, if not explicitly stated, are given in Table III. The time and space units are not specifically defined due to the abstractness of this model.

Trajectories were analyzed by numerically integrating the model equations. Examples of trajectories are given in Fig. 3. Fig. 3(a) shows a simple turn - almost a U-turn. This is a valid solution of the collision avoidance task. Fig. 3(b) shows 
an agent that turned too far. The initial big left turn triggered immediately a right turn which was followed by a wide left turn. By adjusting the decay rate $\lambda$ almost arbitrarily complex trajectories of this class were generated. However, this behavior is not desirable for collision avoidance. This applies to the following two classes as well: periodic behavior shown in Fig. 3(c) and quasi-periodic behavior shown in Fig. 3(d). In both cases, the agent performs a big initial left turn. In Fig. 3(c), this initial turn brings the agent back to a former position $\mathbf{x}$ and the same hormone values leading to periodic behavior. In Fig. 3(d) the agent does not reach the exact same position but is captured in a repetitive trajectory that moves as a whole slowly to the upper right. (a) $\lambda=0.1$

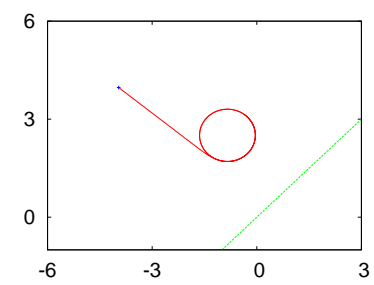

(c) $\lambda=0.005, v=0.08, r=4$

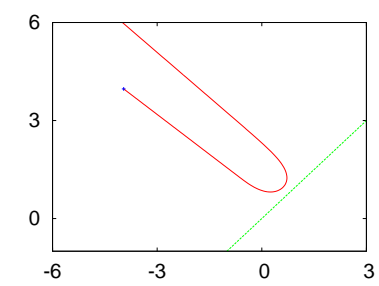

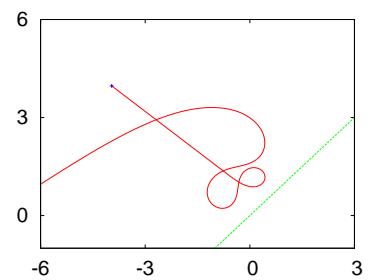

(b) $\lambda=0.01$

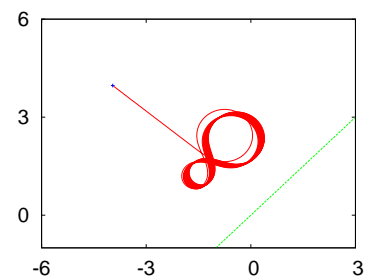

(d) $\lambda=0.0063, v=0.08, r=4$
Fig. 3. Example trajectories of the agent obtained by numerical integration of the mathematical model.

Apparently this mathematical model served only as a tool for preliminary investigations because it is a rather crude abstraction. Complexities, that occur using robotic hardware, such as noisy sensors or variant time steps were not accounted for. Thus, it was not expected to find the reported periodic or quasi-periodic behavior in the robots. Still, this model gives a good overview of possible agent behaviors.

\section{IMPLEMENTATION OF THE CONTROLLER ON THE ROBOT}

The main objective of this work is to test the feasibility of the AHHS on a real robot platform. Performance evaluations and comparisons to other controller types are not part of this work (but see section VI).

To test the abilities of the controller on a robot including the "limitations" of real world problems like noisy sensor input values, we choose an e-puck robot [6] as an implementation platform of the controller. The dimensions of this cylindric, wheeled robot are $5 \mathrm{~cm}$ of height and $7 \mathrm{~cm}$ of diameter. It is equipped with eight IR proximity sensors. For the algorithm only two sensors were used which are pointing $45^{\circ}$ in forward direction.
The arena for the experiments was a square with a side length of $1 \mathrm{~m}$. Four cylindrical obstacles with a diameter of $10 \mathrm{~cm}$ were arranged symmetrically with a distance of $45 \mathrm{~cm}$ from each corner of the square (see Fig. 1).

Preliminary tests were performed to calibrate the values of the parameters of the controllers. Three of the four types of trajectories which were described in the mathematical analysis could also be observed on the real robot: simple turns, overreacted turns and trajectories periodical turns (circular trajectories). The parameters for the experiments were set to values when simple turns were observed. Runs with these parameters were considered to be successful because the robot prevented to touch the wall of the arena or the obstacles for at least 2 min. Results of preliminary tests of finding feasible parameter sets are shown in Tab. I and II.

As explained above, hormone $H_{d}$ is responsible for the default velocity of the robot. The value of base emission rate $\alpha$ was calibrated in that way that the robot was going at about $1 / 5$ of the maximum speed. An optimization for speed was not done.

\section{RESULTS}

\section{A. Controller using one hormone}

The following runs were performed with the parameter values which were calibrated by preliminary tests for this controller type (see Tab. I) ${ }^{1}$. In the experiment the controller was tested in three different situations approaching a wall:

1) wall on the right hand side (angle: $45^{\circ}$ )

2) wall in the frontal center of the robot

3) wall on the right hand side (angle: $45^{\circ}$ )

During these tests the internal values (infrared sensor inputs, the hormones and the actuator control values) of the robot controller were logged. Fig. 4(a) shows the values obtained by the sensor $S_{r}$, the value for $H_{d}$ and, the output of the controller sent to the actuators $A_{r}$, thus the parameter values of the right compartment in a situation when the robot approaches a wall which is on the right hand side. After three time steps the sensor detects the "approaching" wall which is translated via the hormone value to the actuators. This increased speed of the wheel on the right side turns the robot away from the wall and the sensor values decrease with increased distance from the wall (beginning at time step 17). Due to high decay rate the base level of the hormone is reached again very fast.

The activity in the left compartment is shown in Fig. 4(b). Beginning from time step 3 very low hormone value change can be detected because of a small diffusion coming from the right compartment. This diffusion has little influence on the actuator value on this side of the robot. At time step 19 a fluctuation of the sensory input can be seen which leads to an increased hormone value. In time step 20 this fluctuation is translated to a short increase of the left actuator speed.

These small fluctuations in combination with asymmetric left and right sensory values are the only chance for this controller to avoid touching the wall when it approach a

\footnotetext{
${ }^{1}$ for a movie see: http://zool33.uni-graz.at/artlife/ahhs
} 


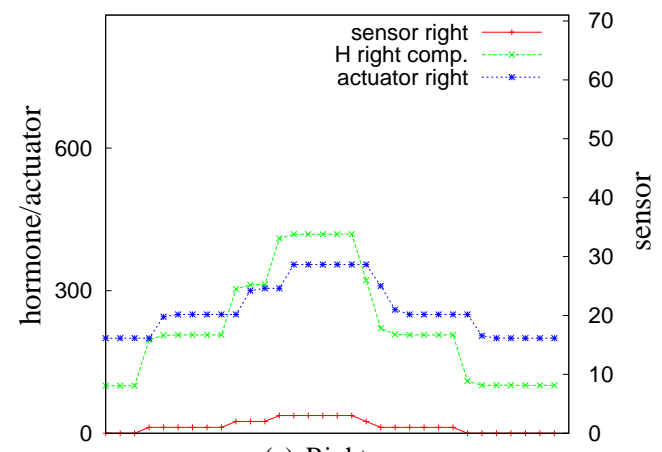

(a) Right

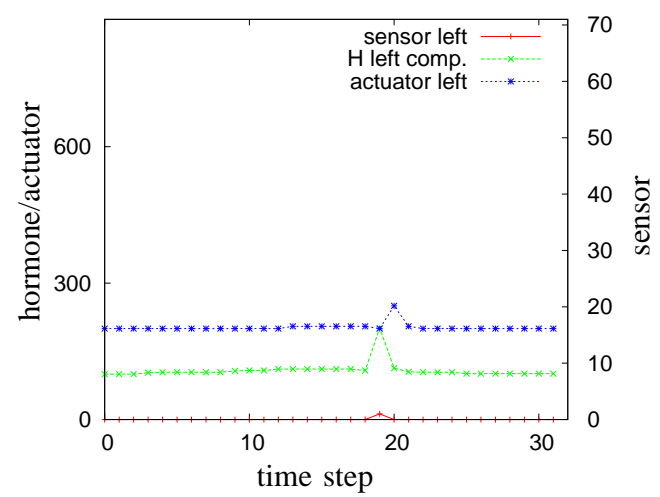

(b) Left

Fig. 4. Temporal progress of the sensor input, hormone, and actuator control with one hormone when approaching a wall to the right.

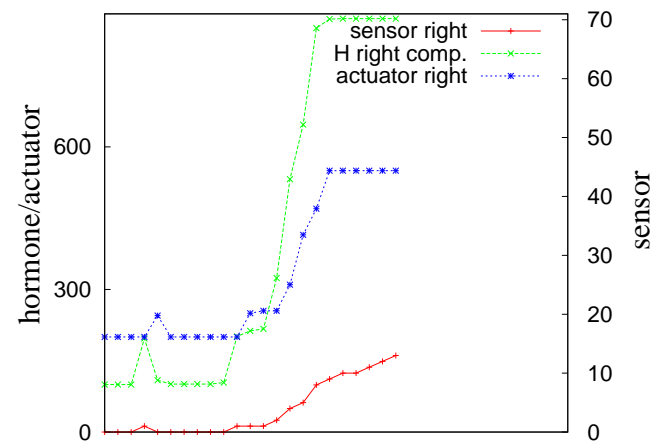

(a) Right

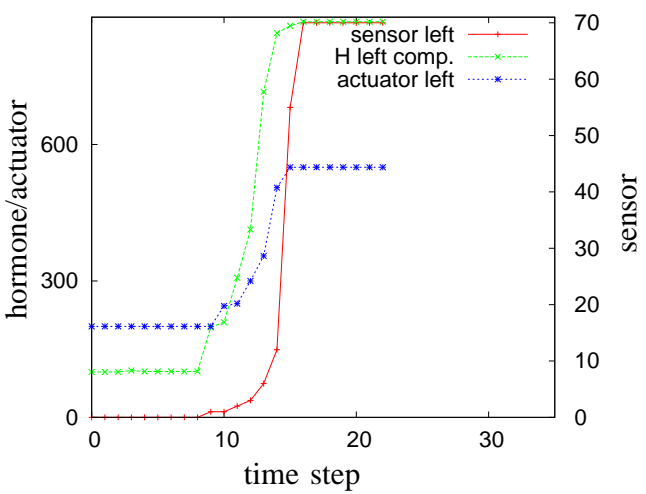

(b) Left

Fig. 5. Temporal progress of the sensor input, hormone, and actuator control with one hormone when head-on approaching a wall. wall frontal centrally. In three of five such cases the robot turns away from the wall. One of the two cases in which the asymmetry is not enough to avoid touching the wall is shown in Fig. 5(a) for the right and in Fig. 5(b) for the left compartment. In both figures the values of the sensors, the hormone and the actuator output increase continuously. Of greater importance is the symmetric increase of the sensory values in Fig. 5(a) and Fig. 5(b) until step number 15. After this point the strong sensory input - which means there is a wall - has no further impact on the steering of the robot, because the maximum of actuator stimulation is reached. This problem can only be omitted if an asymmetric value at the beginning of the approach leads to small turn which is increased automatically every time step.

\section{B. Controller using three hormones}

In comparison to the controller with only one hormone we obtained better results with the controller using three hormones ${ }^{2}$. Especially in the problematic situation of approaching the wall centrally. Fig. 6 and Fig. 7 show the values obtained by the infrared sensors, the values for hormone $H_{r}$ and $H_{l}$, and the output of the controller sent to the actuators. In case of Fig. 6, the robot was positioned such that it approached a wall to its right hand side in an angle of about $45^{\circ}$. Thus, only the right sensor reached values above 0 , while the left sensor and hormone $H_{l}$ were 0 . Hormone $H_{r}$ was produced in the right compartment, see Fig. 6(a), and diffused into the left compartment, see Fig. 6(b). The left wheel slowed down a bit and the right wheel was accelerated leading to a left turn as desired.

In case of Fig. 7, the robot was positioned such that it approached a wall frontally. The collision with the wall was avoided by a big turn to the right in this example. The symmetry was broken by the left sensor reacting two time steps earlier, see Fig. 7(b), than the right sensor, see Fig. 7(a). The left actuator was operated with negative values, that means it was turning backwards. With the right actuator turning forward this lead to a turn on a spot which is a good strategy when centrally approaching a wall. Even if the symmetry breaking would not occur or would occur too late the robot would still avoid the wall. In this case both actuators would be operated with negative values until the distance to the wall is save again.

\section{CONCLUSIONS AND FUTURE WORKS}

We showed that AHHS, as described recently in multiagent simulation systems, can be analyzed well with simple mathematical models [3]. In these models, the parameters that govern the system's dynamics can be studied in detail, and exhaustive parameter sweeps can easily be done with reasonable computational costs. In addition, we demonstrated that AHHS controllers can be implemented on real robotic hardware, and also robots with low computational capabilities (like the e-puck robot) can be controlled by these controllers. Although this article reports only an exemplary

\footnotetext{
${ }^{2}$ for a movie see: http://zool33.uni-graz.at/artlife/ahhs
} 


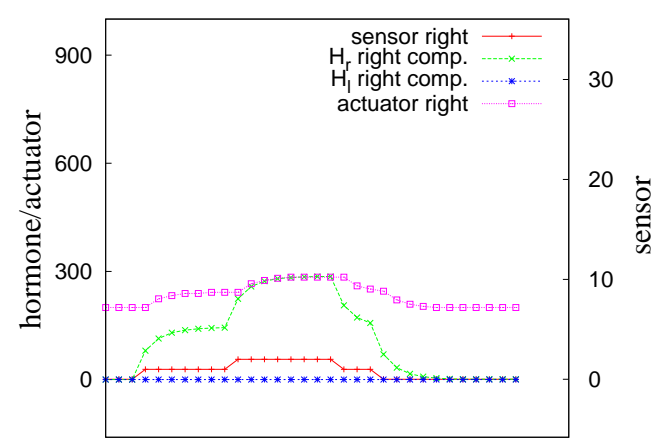

(a) Right

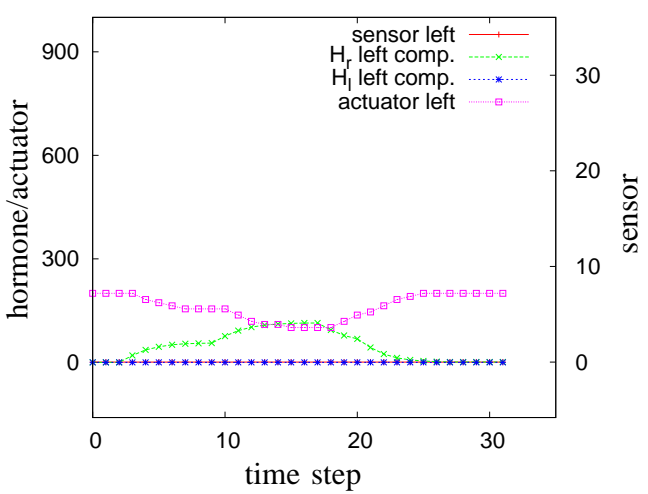

(b) Left

Fig. 6. Temporal progress of the sensor input, hormone, and actuator control with three hormones when approaching a wall to the right.

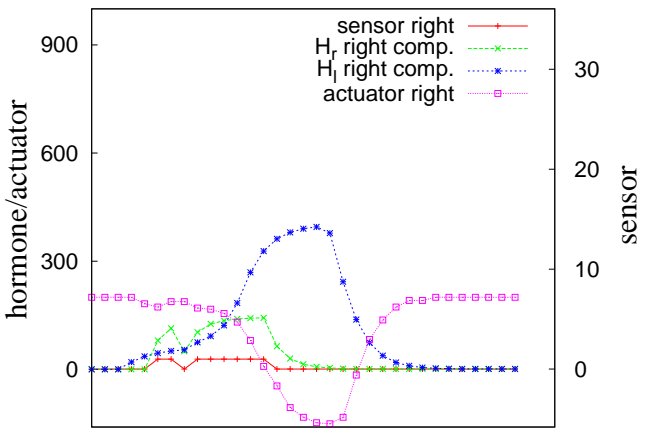

(a) Right

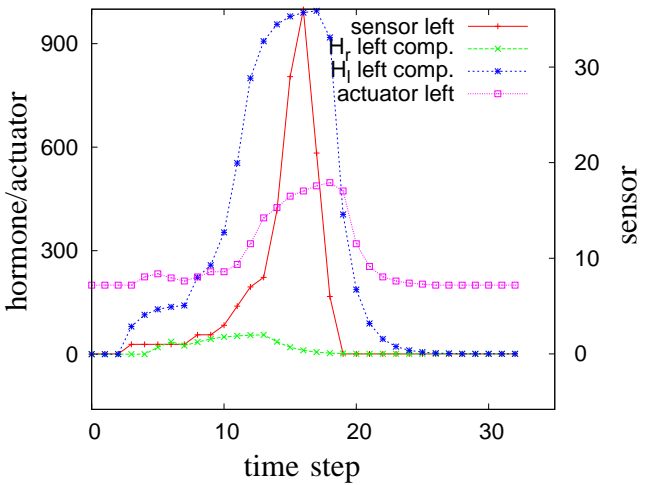

(b) Left

Fig. 7. Temporal progress of the sensor input, hormone, and actuator control with three hormones when head-on approaching a wall. case study on robotic hardware, it gives justified hope that more complex behaviors can be generated on robots with these methods (see [7]). This will be done by successively adding new sensors to the system, which secret additional virtual hormones and interact with the basic driving and collision avoidance system. Thus, a more complex AHHS system can be constructed based on a previously tested one, rather similar to the "subsumption" architecture approach [8]. The most important insight of this study was that the AHHS controller can perform the desired behaviors even in timecritical situations on limited micro processors, that is, the robot was able to avoid collisions.

After showing the feasibility of transferring the AHHS to real robots, the next step of future work is the analysis of the performance and comparison to other controller types like artificial neural networks or stochastic control schemes. Then we will implement more complex AHHS on robotic hardware. One significant extension will be the application of AHHS to swarm robots and on multi-modular robotic systems [7]. In such systems AHHS will be a very interesting control paradigm, as inter-robotic communication will be implemented similar to sensor-induced hormone secretion, thus, mimicking the step from uni-cellular organisms to multi-cellular lifeforms. In addition, we are interested in their computational abilities as well as their evolvability in a mathematical model and in multi-agent systems. Such an evolutionary adaptation can be, in its simplest form, a classical genetic algorithm [9] which operates on the parameters that govern the dynamics of the "hormone flow" inside of an AHHS system. But also adapting the internal structure of the AHHS, that is the topology of the compartments, seems to us to be promising for evolutionary optimization.

\section{ACKNOWLEDGMENTS}

We were supported by the following grants: EU-IST FET project 'SYMBRION', no. 216342, EU-ICT project 'REPLICATOR', no. 216240.

\section{REFERENCES}

[1] D. Floreano, P. Husbands, and S. Nolfi, "Evolutionary Robotics," in Handbook of Robotics, B. Siciliano and K. Oussama, Eds. Berlin: Springer, 2008, ch. 61, pp. 1423-1452.

[2] S. Nolfi and D. Parisi, "Learning to adapt to changing environments in evolving neural networks," Adapt. Beh., vol. 5, no. 1, pp. 75-98, 1997.

[3] T. Schmickl and K. Crailsheim, "Modelling a hormone-based robot controller," in MATHMOD 2009 - 6th Vienna International Conference on Mathematical Modelling, 2009.

[4] F. Poelwijk, D. Kiviet, D. Weinreich, and S. Tans, "Empirical fitness landscapes reveal accessible evolutionary paths," Nature, vol. 445, pp. 383-386, January 2007.

[5] W.-M. Shen, P. Will, A. Galstyan, and C.-M. Chuong, "Hormoneinspired self-organization and distributed control of robotic swarms," Autonomous Robots, vol. 17, pp. 93-105, 2004.

[6] “e-puck desktop mobile robot - website," 2009, http://www.e-puck.org/.

[7] T. Schmickl, J. Stradner, H. Hamann, and K. Crailsheim, "Major feedbacks that support artificial evolution in multi-modular robotics," in Exploring New Horizons in Evolutionary Design of Robots (EvoDeRob) - IROS'09 workshop, ser. LNCS. Springer, (in press).

[8] R. A. Brooks, "Intelligence without representation," Artificial Intelligence, vol. 47, 1991.

[9] J. H. Holland, Adaptation in Natural and Artificial Systems. Cambridge, MA, USA: MIT Press, 1992. 